\title{
NPP VIIRS and Aqua MODIS RSB Comparison Using Observations from Simultaneous Nadir Overpasses (SNO)
}

\author{
A. $\mathrm{Wu}^{\mathrm{a}}$ and $\mathrm{X}$. Xiong ${ }^{\mathrm{b}}$ \\ ${ }^{\text {a}}$ The Sigma Space Corporation, Lanham, MD 20706 \\ ${ }^{\mathrm{b} S}$ Science and Exploration Directorate, NASA/GSFC, Greenbelt, MD 20771
}

\begin{abstract}
Suomi NPP (National Polar-orbiting Partnership) satellite (http://npp.gsfc.nasa.gov/viirs.html) began to daily collect global data following its successful launch on October 28, 2011. The Visible Infrared Imaging Radiometer Suite (VIIRS) is a key NPP sensor. Similar to the design of the OLS, SeaWiFS and MODIS instruments, VIIRS has on-board calibration components including a solar diffuser (SD) and a solar diffuser stability monitor (SDSM) for the reflective solar bands (RSB), a V-groove blackbody for the thermal emissive bands (TEB), and a space view (SV) port for background subtraction. Immediately after the VIIRS nadir door's opening on November 21, 2011, anomalously large degradation in the SD response was identified in the near-IR wavelength region, which was unexpected as decreases in the SD reflectance usually occur gradually in the blue $(\sim 0.4 \mu \mathrm{m})$ wavelength region based on past experience. In this study, we use a well-calibrated Aqua MODIS as reference to track and evaluate VIIRS RSB stability and performance. Reflectances observed by both sensors from simultaneous nadir overpasses (SNO) are used to determine VIIRS to MODIS reflectance ratios for their spectral matching bands. Results of this study provide an immediate post-launch assessment, independent validation of the anomalous degradation observed in SD measurements at near-IR wavelengths and initial analysis of calibration stability and consistency.
\end{abstract}

Keywords: VIIRS, calibration, reflective solar bands, solar diffuser, sensor comparison

\section{INTRODUCTION}

Suomi NPP (National Polar-orbiting Partnership) satellite (http://npp.gsfc.nasa.gov/viirs.html) provides new remote sensing measurements of land, ocean and atmosphere, as a transition from the existing Terra and Aqua Earth-observing missions, and was launched successfully on October 28, 2011. ${ }^{1,2}$ The Visible Infrared Imaging Radiometer Suite (VIIRS) is a key sensor carried on Suomi NPP. ${ }^{3}$ VIIRS is a scanning radiometer and collects visible and infrared imagery and radiometric measurements of Earth's surface. It extends and improves upon a series of measurements initiated by the Advanced Very High Resolution Radiometer (AVHRR) and the Moderate Resolution Imaging Spectroradiometer (MODIS). VIIRS has 22 bands with a spectral range from 400 to $12000 \mathrm{~nm}$. The Earth view swath covers a distance of $3000 \mathrm{~km}$ over scan 
angles of $\pm 56.0^{\circ}$ off nadir, achieved though a constant-rate rotating telescope assembly (RTA) and a double sided half angle mirror (HAM) rotating at half the speed of the RTA. Similar to the design of the OLS, SeaWiFS and MODIS instruments, VIIRS has on-board calibration components including a solar diffuser (SD) and a solar diffuser stability monitor (SDSM) for the reflective solar bands (RSB), a V-groove blackbody for the thermal emissive bands (TEB), and a space view (SV) port for background subtraction.

Immediately after VIIRS nadir door's opening on November 21, 2012, VIIRS started to produce the sensor data record (SDR) for VIS and near-IR bands. Data collection for S/MWIR bands and the rest of the TEB did not start until the instrument cryoradiator was activated. Therefore, it was possible to conduct an immediate post-launch assessment of the stability and performance of most of the VIIRS RSB. This is particularly important to support the VIIRS RSB on-orbit calibration since application of on-orbit calibration coefficients depends on trending analysis of the data obtained from onboard calibrators.

A comparison of VIIRS RSB measurements with those from a stable and well-calibrated sensor provides a quick and proper assessment of its post-launch stability and performance. Major challenges in cross-sensor comparison exist in how to obtain high quality data sets due to the effects of different observation times, viewing and illumination angle differences and sensor-tosensor spectral response differences. Observations from simultaneous nadir overpasses (SNO) between two polar orbiting satellites significantly reduce the biases caused by the viewing geometry and observation time differences. ${ }^{4}$ This approach has been successfully applied to compare pairs of succeeding NOAA -7 to 11 AVHRR for both visible and infrared channels, ${ }^{5}$ infrared channels of the High Resolution Infrared Radiation Sounders (HIRS) onboard NOAA15, -16 and $-17^{6}$ and MODIS onboard Terra and Aqua spacecrafts for both RSB and TEB. ${ }^{7}$

This study applies the same SNO approach ${ }^{4-7}$ to compare Suomi NPP VIIRS and Aqua MODIS for their spectrally matched RSB (Table 1). The reason that Aqua MODIS is selected is because it has a more stable performance and longer SNO periods than Terra MODIS (both in ascending orbit). The VIIRS performance evaluation is conducted with three goals: 1) immediate postlaunch performance assessment, 2) degradation trends and, 3) calibration stability and consistency. The latter two assessment results are based on the course of the first half year's VIIRS on-orbit operation.

Table 1. List of central wavelength (CW) and band width (BW) for VIIRS/MODIS (V/M) spectrally matched RSB in the visible and near-infrared wavelength region (units in nm)

\begin{tabular}{|c|c|c|c|c|c|c|c|c|c|}
\hline V/M & M1/B8 & M2/B9 & M3/M10 & M4/B4 & M5/B1 & M6/B15 & M7/B2 & I1/B1 & I2/B2 \\
\hline CW & $412 / 412$ & $445 / 443$ & $488 / 488$ & $555 / 555$ & $672 / 645$ & $746 / 748$ & $865 / 858$ & $640 / 645$ & $865 / 858$ \\
\hline BW & $20 / 15$ & $18 / 10$ & $20 / 10$ & $20 / 20$ & $20 / 50$ & $15 / 10$ & $39 / 35$ & $80 / 50$ & $39 / 35$ \\
\hline
\end{tabular}




\section{SNO APPROACH}

The SNO time and location between MODIS and VIIRS can be calculated using the NORAD two-line element SGP4 propagation model. ${ }^{8}$ The model can be applied to any pair of two polar orbiting satellites. In our early studies, the same technique was used to get the SNO time and location between MODIS and AVHRR ${ }^{7}$ and other polar orbiting sensors. ${ }^{9}$ In the case of Aqua MODIS and NPP VIIRS, the SNO orbits are nearly parallel, providing SNO periods for 20 to 50 minutes depending on the orbit (Figure 1). On the other hand, SNO periods between Terra MODIS and NPP VIIRS are rather short due to their non-parallel orbits (Figure 1). This is one of the important reasons that we selected to use data from Aqua MODIS and NPP VIIRS SNO orbits for this cross-sensor comparison study.

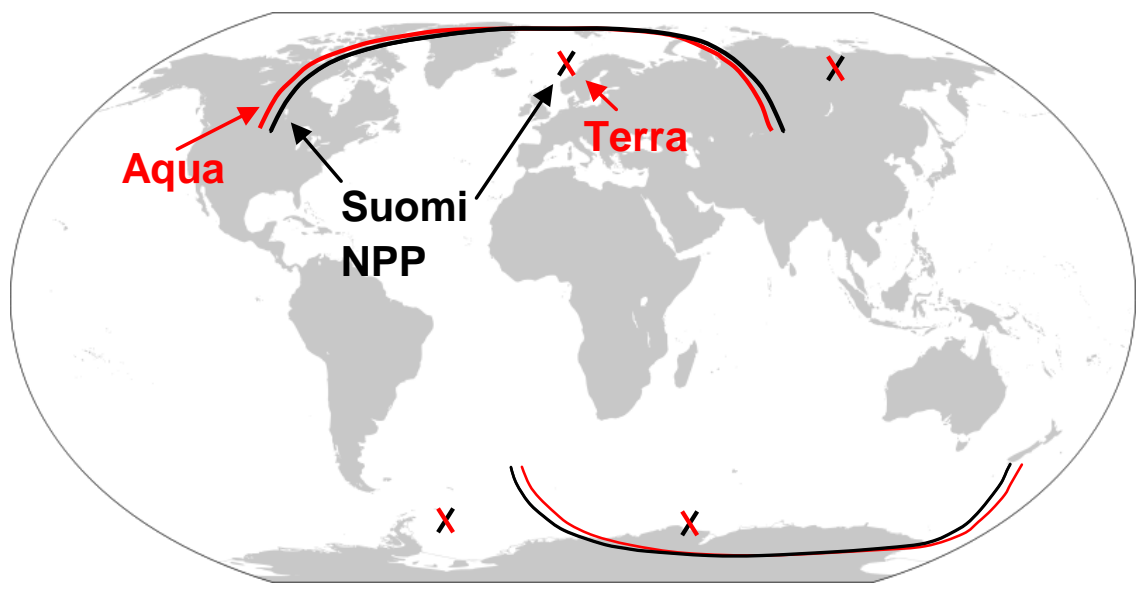

Figure 1. Diagram of Aqua (red) and Suomi NPP (black) SNO orbits. Also shown are SNO orbits between Terra and Suomi NPP.

In order to achieve high quality inter-comparisons, as discussed in our previous studies, ${ }^{7,9} \mathrm{SNO}$ samples are collected over a near nadir area within $\pm 5^{\circ}$ off-nadir for both MODIS and VIIRS. The selection of the SNO area is limited to high latitude regions $\left(\sim 70^{\circ} \mathrm{S} / \mathrm{N}\right)$ so the nadir distances of both sensors are relatively closer (Figure 1). Then, a pixel-by-pixel co-location between MODIS and VIIRS data sets is performed over the selected area. VIIRS nadir spatial resolution is $0.371 \mathrm{~km}$ for I bands and 0.742 for $\mathrm{M}$ bands, compared with three different nadir resolutions of $0.25,0.50$ and $1.0 \mathrm{~km}$ for MODIS bands. Thus, on the pixel level, the VIIRS and MODIS footprints are quite different. To reduce the impact of the footprint mismatch, relatively homogeneous surface areas are preferred. Another option is to co-locate pixels to large grid areas such as $1^{\circ}$ of latitude by $1^{\circ}$ of longitude. Since co-location to large grid areas only works for nonsaturated bands, this approach cannot be applied to MODIS ocean color bands (bands 8-10, 15). The comparison presented in this study is still based on the pixel-by-pixel co-location. We limited our selected areas to relatively homogeneous surfaces by excluding areas with large 
standard errors in VIIRS to MODIS radiance or reflectance ratios. To further reduce variations in comparison data sets, an average over 20 points is calculated for both sampled VIIRS and MODIS SNO pixels.

\section{DIFFERENCES IN RSR AND SOLAR IRRADIANCE MODELS}

Differences in the relative spectral response (RSR) need to be considered for cross-sensor comparison. Between VIIRS and MODIS RSB, the RSR differences between the spectrally matched bands (Table 1) are generally small except between VIIRS M5 and MODIS band 1 (Figure 2). Replacing MODIS band 1 with either band 13 or 14 is better in terms of spectral match but the saturation issue in either band (13 or 14) would provide a much lower number of valid data points than the use of band 1 .

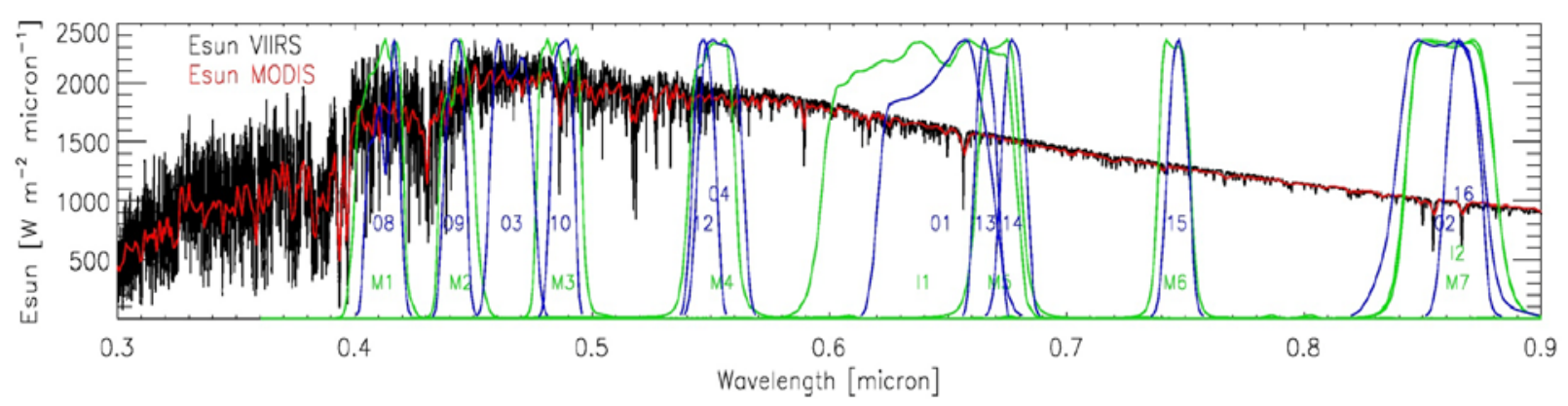

Figure 2. VIIRS and MODIS solar irradiance (i.e., Esun) models and their visible/nearinfrared band RSR.

Comparison of the solar irradiance model used in the L1B product between VIIRS and MODIS is also important to understand if additional differences in radiance or reflectance are due to the use of a different solar irradiance model. This is because the calibration of VIIRS RSB is based on radiance measurements and MODIS is based on reflectance measurements. Thus, comparison for either radiance or reflectance involves using a solar irradiance model. Figure 2 shows that the two solar irradiance models match well at wavelengths longer than $0.55 \mu \mathrm{m}$. At wavelengths shorter than $0.55 \mu \mathrm{m}$, however, the latest VIIRS solar irradiance model is much higher in spectral resolution and provides a more detailed description of solar spectral variations. To further investigate the impact of the two solar irradiance models, a RSR integrated solar irradiance ratio between VIIRS and MODIS spectrally matched bands (Table 1) is calculated. Figure 3 compares the ratios obtained using the VIIRS and MODIS based solar irradiance models. The results show that using either solar model produces very close ratios for all spectrally matched VIIRS and MODIS bands. Our original calculation results, however, show that the ratios for M1/Band8 and M2/Band9 have differences of up to 4\% between using the two solar models. Such differences, however, are likely due to a computational error in the use of the VIIRS based solar irradiance model. This is because there is a significant spectral resolution 
mismatch between the VIIRS solar irradiance model and MODIS RSR. A direct calculation of the band spectrally-integrated radiance with both data sets would cause large errors due to a low spectral resolution in the MODIS RSR. To reduce such computational errors, a smoothing spline fit is applied to the VIIRS solar irradiance model. The ratio values are generally between 0.98 and 1.02 depending on band and the particularly low ratio values of around 0.95 for M5/band1 is due to a significant RSR difference between the two bands (Figure 2).

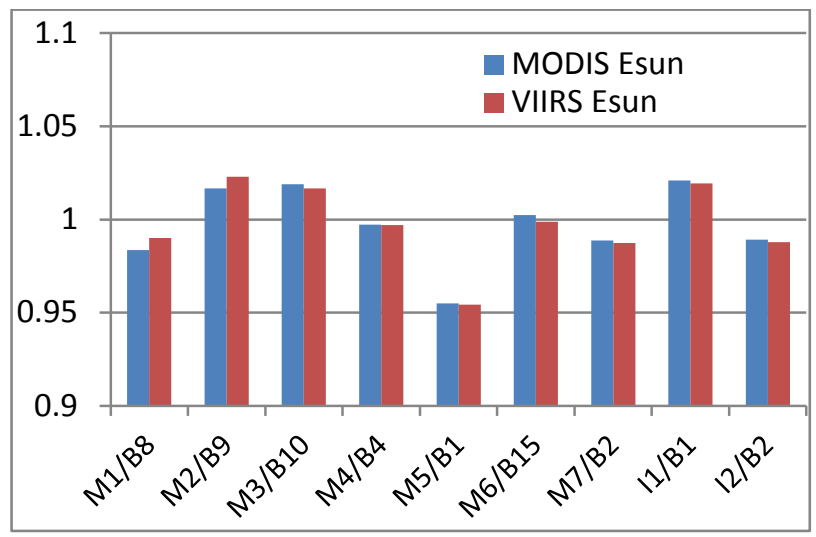

Figure 3. VIIRS to MODIS radiance ratios derived from two separate sensor-based Esun models (MODIS and VIIRS) for their spectrally matched bands in the visible/near-infrared wavelength range.
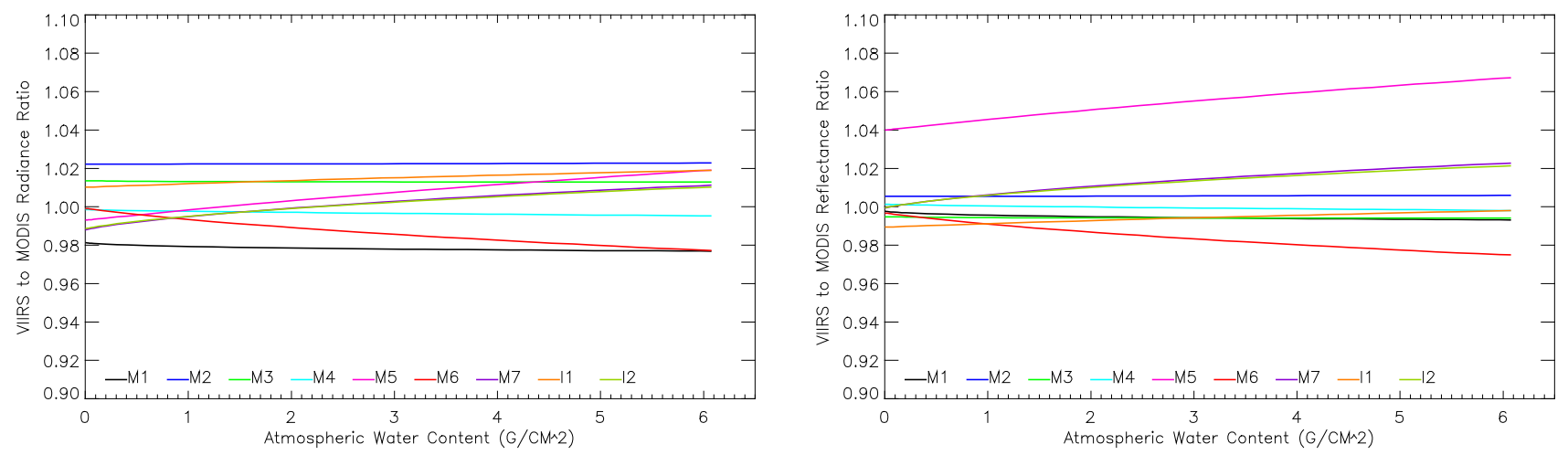

Figure 4. VIIRS to MODIS radiance (left) and reflectance (right) ratios derived from MODTRAN5 simulated at-sensor radiances as a function of atmospheric water content.

Theoretical radiance ratios between VIIRS and MODIS spectrally matched bands (Table 1) can be simulated using MODTRAN (MODerate resolution atmospheric TRANsmission) providing visible/near-infrared spectral radiance results. In this study, the most recently released version of 
the code, MODTRAN5, ${ }^{10}$ is used to produce simulated at-sensor radiances. Input options include a standard atmospheric profile (U.S. Standard Atmosphere 1976), a fixed viewing and illumination geometry, a wide range of atmospheric water content, a standard aerosol model, cloud-free conditions, and surface reflectance. Outputs are specified for at-sensor radiances from 350 to $2500 \mathrm{~nm}$ with a spectral resolution of $0.5 \mathrm{~nm}$.

Given a simulated at-sensor radiance model, a RSR integrated band radiance can be computed. Figure 4 plots the simulated VIIRS to MODIS radiance and reflectance ratios as a function of atmospheric water content. Results show that the radiance ratios are between 0.98 and 1.02 over a wide range of water content $\left(0.0\right.$ to $\left.6.0 \mathrm{gcm}^{-2}\right)$. For the reflectance ratios, similar results are obtained except for M5 where the ratios are between 1.04 and 1.06 due to a large RSR difference between VIIRS and MODIS (Figure 2). The impact on the ratios due to water vapor is within $2 \%$. Figure 4 indicates that if both VIIRS and MODIS are perfectly calibrated, radiances or reflectances from both sensors should have agreement of within 2\% (except for M5 in the reflectance comparison).

\section{VIIRS AND MODIS RSB COMPARISON}

\subsection{Immediate post-launch assessment}

VIIRS nadir door was successfully opened at 16:03 GMT on November 21, 2011. Right after the nadir door's open, the VIIRS RSB started to work normally except for S/MWIR bands and the rest of TEB which required the cryoradiator to be activated. The first SNO event started at 20:54 GMT on the same day (November 21), which was less than 5 hours after the nadir door was opened. Analysis of data collected from the first SNO event provides a good opportunity to perform an immediate post-launch assessment for the calibration of the RSB. Figure 5 shows a one-to-one comparison between VIIRS and MODIS reflectances for a few RBS using the SNO data collected on November 21, 2012. There is an excellent correlation between the two indicating that VIIRS RSB detectors were working as expected. Values of the reflectance ratios are $0.95,1.07,0.12$ and 1.19 respectively for the four bands shown in the figure. These ratios are quite different from the theoretical results shown in Figure 4, where ratios between 0.98 and 1.02 are expected. Since the ratios from Figure 5 are either much lower or higher than 1.0, it is very unlikely they are due to a rapid degradation in RSB. Given the fact that the period between the launch of Suomi NPP and VIIRS nadir door's opening was less than one month, there should not be any noticeable degradation of the VIIRS RSB detectors or HAM mirrors. Any degradation of the VIIRS SD, however, has no direct impact on the reflectance, since no on-orbit calibration of the RSB was applied during the first few months after the nadir door's opening. This raised concerns over whether the VIIRS RSB pre-launch calibration coefficients were correct or they were implemented correctly in the SDR (sensor data record) code. It was later confirmed that the first SDR look up tables (LUT) for RSB were implemented incorrectly. The analysis results 
shown in this study, along with other independent results, helped to provide an immediate postlaunch assessment of VIIRS SDR data quality and consistency.
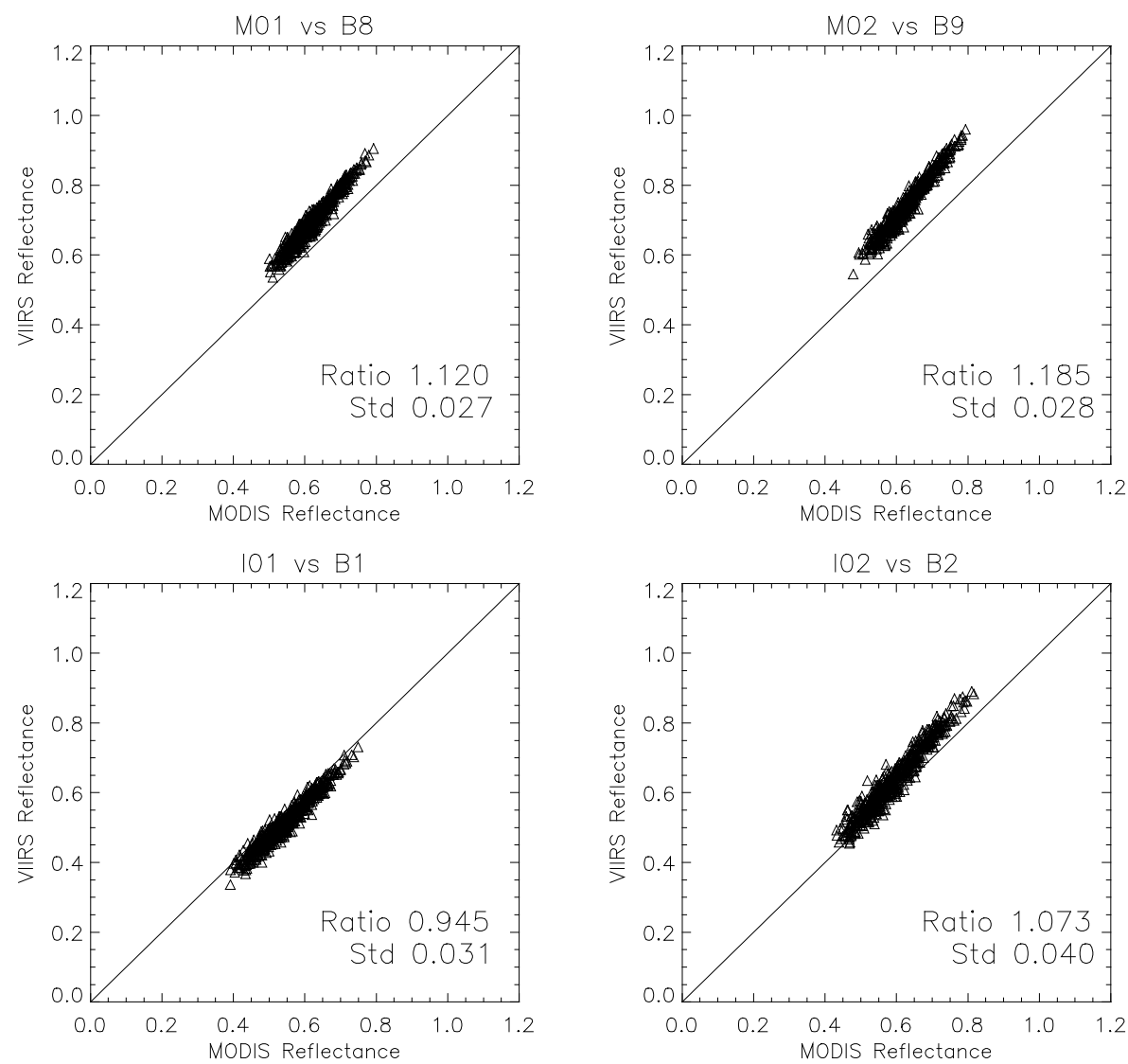

Figure 5. One-to-one comparison of reflectance between VIIRS and MODIS spectrally matched bands obtained from the first SNO event on November 21, 2011. Also shown are the mean and standard error of VIIRS to MODIS reflectance ratios.

\subsection{Validation of degradation anomaly}

The degradation of VIIRS RSB is tracked by on-orbit measurements of detector responses to the sun-light illuminated SD. A scaling factor, F, depending on band, detector, HAM side and subsample is provided after the SD calibration in each orbit, currently calculated offline. Applying $F$ factors after the detector raw response corrected with pre-launch based calibration coefficients produces the SDR radiance/reflectance product.

Trending results of $F$ show that there is a significant degradation in the $600-1000 \mathrm{~nm}$ region (see related studies presented in this conference). Such degradation contradicts previous experience on the SD on-orbit performance. Since up to $10 \%$ degradation in $F$ was observed within the first 
month after the nadir door's opening, it triggered an immediate anomaly investigation into the accelerated and continued degradation of VIIRS M5 to M7 and I2 (670 - $865 \mathrm{~nm})$. To support the anomaly investigation, trends of VIIRS to MODIS reflectance ratios were very helpful in validating the observed degradations in $F$ from an independent data source.

Figure 6 shows trends of VIIRS to MODIS reflectance ratios for M1, M5 and I2, normalized to day 1 (November 21, 2011). The trends are derived from SNO events that occur once every three days. No on-orbit calibration is applied to determine VIIRS reflectances, so the anomaly driven degradation can be easily seen in the trends. The trending results indicate that I2 (865 nm) has a more than 20\% degradation during the first six months, compared with 5\% for M5 (672 nm) and $2 \%$ for M1 $(412 \mathrm{~nm})$. We also plot the $F$ factor trends along with the normalized ratios and they are in reasonably good agreement.

The testing of the VIIRS witness samples by other investigation teams revealed that there was an unusual compound on the surface of the VIIRS mirror--Tungsten Oxide $\left(\mathrm{WO}_{3}\right) .{ }^{11}$ The VIIR mirror immediately began to degrade when exposed to UV. The reflectance test following a 24 hour exposure to full sun showed significant degradation in the $600-1000 \mathrm{~nm}$ range. Although the anomaly is unique to VIIRS, the results in Figure 6 indicate that $F$ factor trends, if applied in a timely fashion, should be able to correct for the anomaly impact on the SDR product.

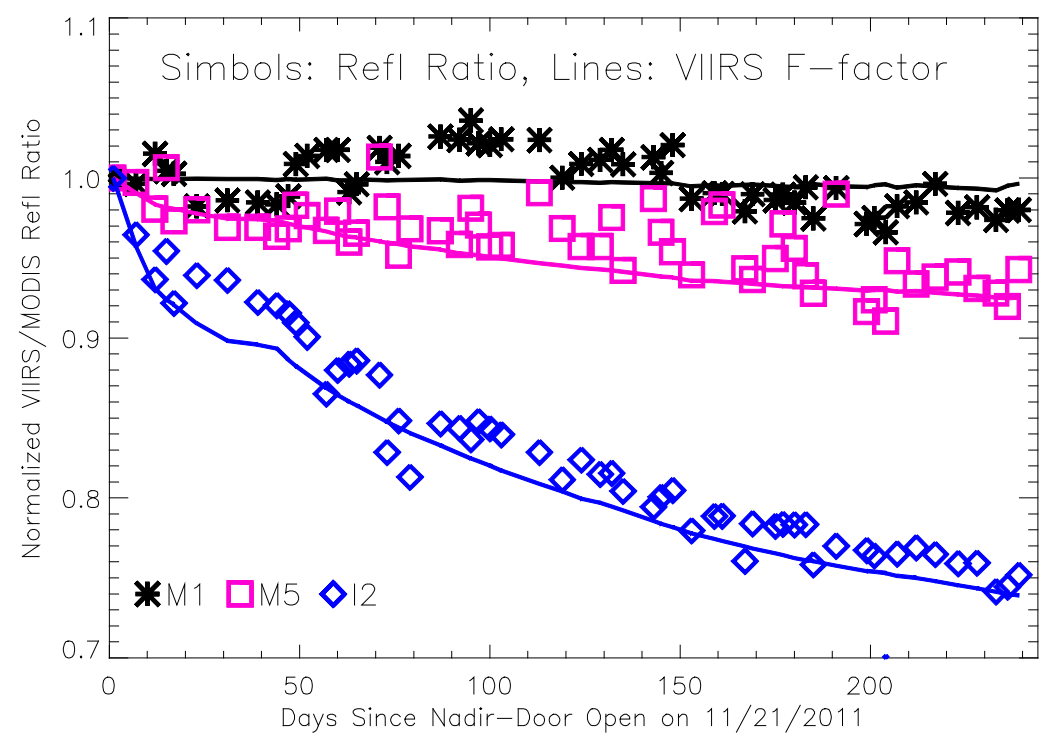

Figure 6. Trends of VIIRS to MODIS reflectance ratios for M1, M5 and I2, derived from VIIRS prelaunch-based calibration coefficients and normalized to the first point. Also shown are VIIRS F factor trends for the same bands. 


\subsection{Calibration stability and consistency}

VIIRS SDR LUT obtained from on-orbit SD measurements was implemented on February 6, 2012 and had been updating since then on a weekly basis. Figure 7 provides the trends of VIIRS to MODIS radiance and reflectance ratios obtained from the processed SNO events. The VIIRS data is based on the SDR product and data before the LUT implementation date (February 6, 2012) was reprocessed offline by applying the $F$ factor correction. Trending results indicate that the calibration of the VIIRS RSB is stable and consistent. The $F$ factor correction is able to correct the VIIRS anomaly caused degradation in the $670-865 \mathrm{~nm}$ range, which is expected as shown in Figure 6.

The ratios in Figure 7 are derived using current MODIS Collection 5 (C5) L1B product. Since Aqua MODIS Collection 6 (C6) has just finished reprocessing for the whole mission, it is important that the latest MODIS L1B is used for a better comparison between VIIRS and MODIS. Because the major MODIS RSB C6 changes are in the two shortest wavelength bands at $412 \mathrm{~nm}$ (band 8) and $443 \mathrm{~nm}$ (band 9), replacing MODIS C5 with C6 would have major impacts on our comparison for VIIRS M1 and M2 (Table 1). Table 2 lists relative percentage differences between VIIRS and MODIS (C5 and C6) and associated uncertainties determined from the SNO trending data. For the comparison in radiance, the two sensor results agree of within $\pm 2 \%$. For reflectance, the same agreement is observed except for M5 and M1. MODIS C6 increases the radiance/reflectance values of bands 8 and 9 by about $4 \%$ and $2 \%$, respectively. Thus, replacing C5 with C6 shows the similar changes in the VIIRS and MODIS comparison for M1 and M2. For other bands, the impact due to the C6 update is very small, as shown in Table 2. The exceptionally large uncertainties for M6 (over 5\%) are mainly due to the fact that the spectrally matched MODIS band 15 saturates over even slightly bright targets and unsaturated pixels correspond to low end of the VIIRS dynamic range.

To further investigate the relative differences between VIIRS and MODIS, the measured ratios in Figure 7 are compared with the theoretical ratios in Figure 4. We selected the theoretical ratios

corresponding to an atmospheric water content of $1.0 \mathrm{gcm}^{-2}$ to represent dry atmospheres for the SNO data collected in high latitude regions. Table 2 lists the MODTRAN simulated theoretical differences between VIIRS and MODIS. The comparison indicates that using MODIS C6 significantly reduces the differences between MODIS band 8 and VIIRS M1 and produces result in closer agreement with the theoretical values. Based on our current simulations and the use of MODIS C6, it is shown that the relative differences between the two sensors are within 2\% for the selected spectrally matched RSB bands. 

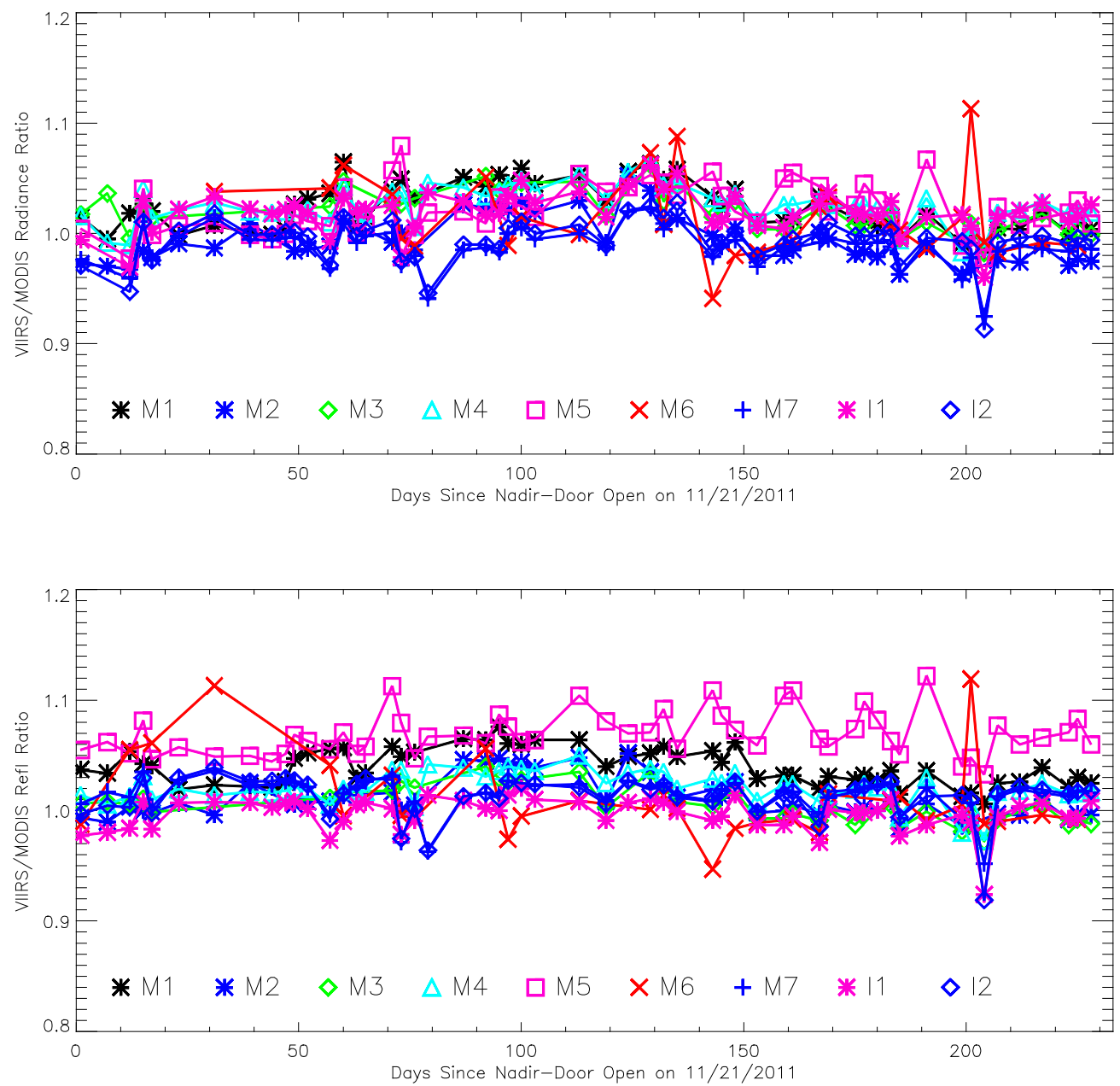

Figure 7. Trends of VIIRS to MODIS ratios in radiance (top) and reflectance (bottom) for their spectrally matched visible and near-infrared bands obtained from their current L1B data product.

Table 2. Means and standard errors of the VIIRS to MODIS ratio in radiance and reflectance for their spectrally matched visible and near-infrared bands. The ratios are determined from VIIRS SDR and MODIS current (C5) and future (C6) version of L1B. Also shown are the ratios derived from combining MODTRAN5 simulated at-sensor radiances with the sensor-dependent RSR and Esun model.

\begin{tabular}{|c|c|c|c|c|c|c|c|c|c|}
\hline & \multicolumn{10}{|c|}{ Radiance } \\
\hline Band & M1 & M2 & M3 & M4 & M5 & M6 & M7 & I1 & I2 \\
\hline C5 & $2.4 \pm 2.1$ & $-0.5 \pm 2.1$ & $2.1 \pm 1.8$ & $2.6 \pm 1.7$ & $2.2 \pm 2.2$ & $1.6 \pm 5.7$ & $-1.2 \pm 1.8$ & $1.9 \pm 1.9$ & $-0.6 \pm 2.0$ \\
\hline C6 & $-1.6 \pm 2.0$ & $-1.3 \pm 2.1$ & $2.9 \pm 1.9$ & $2.9 \pm 1.7$ & $2.4 \pm 2.3$ & $2.2 \pm 5.3$ & $-1.0 \pm 1.6$ & $2.1 \pm 1.8$ & $-0.5 \pm 1.7$ \\
\hline MODTRAN & -2.1 & 2.2 & 1.3 & -0.2 & -0.2 & -0.7 & -0.5 & 1.2 & -0.5 \\
\hline
\end{tabular}




\begin{tabular}{|c|c|c|c|c|c|c|c|c|c|}
\hline & \multicolumn{10}{|c|}{ Reflectance } \\
\hline Band & M1 & M2 & M3 & M4 & M5 & M6 & M7 & I1 & I2 \\
\hline C5 & $4.0 \pm 1.6$ & $1.2 \pm 1.8$ & $0.7 \pm 1.6$ & $2.1 \pm 1.4$ & $6.9 \pm 1.9$ & $2.1 \pm 6.3$ & $1.4 \pm 1.6$ & $-0.3 \pm 1.5$ & $1.3 \pm 1.9$ \\
\hline C6 & $-0.2 \pm 1.6$ & $0.2 \pm 1.9$ & $1.3 \pm 1.7$ & $2.3 \pm 1.3$ & $6.9 \pm 2.1$ & $2.4 \pm 6.1$ & $1.4 \pm 1.4$ & $-0.3 \pm 1.2$ & $1.3 \pm 1.5$ \\
\hline MODTRAN & -0.4 & 0.6 & -0.6 & 0.0 & 4.6 & -0.9 & 0.6 & 0.9 & -1.3 \\
\hline
\end{tabular}

\section{SUMMARY}

In this study, a well-calibrated Aqua MODIS is used as reference to track and evaluate the RSB stability and performance for the newly launched Suomi NPP VIIRS. VIIRS to MODIS ratios in radiance and reflectance from SNO events once every three days are calculated for spectrally matched bands. The VIIRS performance evaluation is conducted for immediate post-launch assessment, degradation anomaly in the $600-1000 \mathrm{~nm}$ wavelength region and calibration stability and consistency. The results of this study indicate that the VIIRS to MODIS comparison based on SNO data is able to provide immediate assessment that the first SDR RSB LUT were implemented incorrectly and confirm that the degradation anomaly occurred in both the SD and earth view sectors. Trending results show that the calibration of the VIIRS RSB is stable and consistent after the on-orbit $F$ factor correction is applied. Finally, with the help of MODTRAN simulated at-sensor radiances, the results of this study show that the relative differences between the two sensors are within $2 \%$ for the selected spectrally matched RSB bands.

\section{ACKNOWLEDGMeNT}

The authors are grateful to colleague Boryana Efremova for her computational assistance and Ning Lei and Jon Fulbright for their provided VIIRS SD/SDSM degradation results.

\section{REFERENCES}

[1] "National Polar-orbiting Operational Environmental Satellite Systems (NPOESS) Preparatory Project (NPP) Instrument Calibration and Support Element Design Document,” NASA/GSFC, unpublished (2008).

[2] Bufler, J., X. Xiong, H. Oudrari, C. Pan, and J. Gleason, "NASA Calibration and Characterization in the NPOESS Preparatory Project (NPP)”, IGARSS, (2009).

[3] Welsch, C., H. Swenson, S.A. Cota, F. Deluccia, J. M. Hass, C. Schueler, R.M. Durham, J.E. Clement, and P.E. Ardanuy, "VIIRS (Visible Infrared Imager Radiometer Suite): A Next-Generation Operational Environmental Sensor for NPOESS”, Intl. Geosciences and Remote Sensing Symposium Proceedings, (2001). 
[4] Cao, C., M. Weinreb, and H. Xu, "Predicting simultaneous nadir overpasses among polar-orbiting meteorological satellites for intersatellite calibration of radiometers," Journal of Atmospheric and Oceanic Technology, vol. 21, pp. 537-542, (2004).

[5] Ciren, P., C. Cao , and J.T. Sullivan, "Consistency in the long-term environmental measurements with NOAA Advanced Very High Resolution Radiometer,” Proceedings of SPIE - Remote Sensing and Modeling of Ecosystems for Sustainability III, 6298, pp. 629810-1-12, (2006).

[6] Cao, C., H. Xu, J. Sullivan, L. McMillin, P. Ciren, and Y. Hou, "Intersatellite radiance biases for the High Resolution Infrared Radiation Sounders (HIRS) onboard NOAA-15, -16, and -17 from simultaneous nadir observations”, Journal of Atmospheric and Oceanic Technology, 22, No. 4, pp. 381-395, (2005).

[7] Wu, A., X. Xiong, and C. Cao, "Terra and Aqua MODIS Inter-comparison of Three Reflective Solar Bands Using AVHRR Onboard the NOAA-KLM Satellites," Int. J. Remote Sens., vol. 29, no. 7, pp. 1997-2010, (2008).

[8] Kelso, T. S. "Validation of SGP4 and IS-GPS-200D Against GPS Precision Ephemerides." Paper AAS 07-127 presented at the AAS/AIAA Spaceflight Mechanics Conference. Sedona, Arizona, (2007)

[9] Wu, A., X. Xiong, H. Murakami, and W. Barnes, "Inter-comparison of Aqua and Terra MODIS using Adeos-II GLI orbital intersections”, Proc. SPIE, Earth Observing Systems X, vol. 5882, no. 58820X, (2005).

[10] Berk, A., G.P. Anderson, P.K. Acharya, E.P. Shettle, MODTRAN 5.2.1 USER'S MANUAL, Air Force Research Laboratory, Space Vehicles Directorate, Air Force Materiel Command Hanscom AFB, MA, (2008).

[11] "Early Assessment of Suomi-NPP VIIRS Radiometric Performance”, NASA VIIRS Calibration Support Team, VIIRS Cal/Val Workshop, April 17, (2012). 